\title{
Energy-Spread Preservation and High Efficiency in a Plasma-Wakefield Accelerator
}

\author{
C. A. Lindstrøm $\odot,{ }^{1, *}$ J. M. Garland, ${ }^{1}$ S. Schröder $\odot,{ }^{1,2}$ L. Boulton, ${ }^{1,3,4}$ G. Boyle, ${ }^{1}$ J. Chappell, ${ }^{5}$ R. D’ Arcy, ${ }^{1}$ P. Gonzalez, ${ }^{1,2}$ \\ A. Knetsch $\odot,{ }^{1, \dagger}$ V. Libov, ${ }^{1}$ G. Loisch $\odot,{ }^{1}$ A. Martinez de la Ossa, ${ }^{1}$ P. Niknejadi $\odot,{ }^{1}$ K. Põder, ${ }^{1}$ L. Schaper, ${ }^{1}$ B. Schmidt,${ }^{1}$ \\ B. Sheeran, ${ }^{1,2}$ S. Wesch $\odot,{ }^{1}$ J. Wood $\odot,{ }^{1}$ and J. Osterhoff ${ }^{1}$ \\ ${ }^{1}$ Deutsches Elektronen-Synchrotron DESY, Notkestraße 85, 22607 Hamburg, Germany \\ ${ }^{2}$ Universität Hamburg, Luruper Chaussee 149, 22761 Hamburg, Germany \\ ${ }^{3}$ SUPA, Department of Physics, University of Strathclyde, Glasgow, United Kingdom \\ ${ }^{4}$ The Cockcroft Institute, Daresbury, United Kingdom \\ ${ }^{5}$ University College London, London, United Kingdom
}

(Received 21 July 2020; revised 5 November 2020; accepted 8 December 2020; published 6 January 2021; corrected 1 February 2021)

\begin{abstract}
Energy-efficient plasma-wakefield acceleration of particle bunches with low energy spread is a promising path to realizing compact free-electron lasers and particle colliders. High efficiency and low energy spread can be achieved simultaneously by strong beam loading of plasma wakefields when accelerating bunches with carefully tailored current profiles [M. Tzoufras et al., Phys. Rev. Lett. 101, 145002 (2008)]. We experimentally demonstrate such optimal beam loading in a nonlinear electron-driven plasma accelerator. Bunches with an initial energy of $1 \mathrm{GeV}$ were accelerated by $45 \mathrm{MeV}$ with an energytransfer efficiency of $(42 \pm 4) \%$ at a gradient of $1.3 \mathrm{GV} / \mathrm{m}$ while preserving per-mille energy spreads with full charge coupling, demonstrating wakefield flattening at the few-percent level.
\end{abstract}

DOI: 10.1103/PhysRevLett.126.014801

Plasma wakefields [1] driven by intense particle beams $[2,3]$ can provide accelerating gradients in the multi-GV/m range [4-6], promising more compact accelerators for high energy physics and photon science [7-10]. Delivering bunches with low energy spread is a key requirement for realizing high-brilliance free-electron lasers [11] and high luminosity with a narrow energy spectrum in linear colliders [12]. Simultaneously, high energy-transfer efficiency is crucial for minimizing the energy consumption of such machines. The solution to both of these problems is strong beam loading, where the presence of a high-current trailing bunch changes the trajectory of in-flowing plasmawake electrons expelled by the driver, thereby altering the longitudinal wakefield and efficiently extracting the kinetic energy of the wake [13-16]. By precisely shaping the current profile of this trailing bunch, the longitudinal wakefield can be locally flattened such that all particles experience the same accelerating gradient. Beam loading has already been experimentally demonstrated in a beamdriven plasma accelerator [17,18], resulting in high energytransfer efficiency (up to $30 \%$ ), but so far with relatively large energy spreads compared to the energy gain. To reach

Published by the American Physical Society under the terms of the Creative Commons Attribution 4.0 International license. Further distribution of this work must maintain attribution to the author(s) and the published article's title, journal citation, and DOI. the sub-percent-level energy spread required for applications, the wakefield must be shaped with a similar level of precision. Tzoufras et al. [19] showed that in the nonlinear regime [20,21] optimal beam loading requires the use of trailing bunches with a trapezoidal current profile, precisely tailored according to the bunch location and the strength of the wakefield. Nontrapezoidal (e.g., Gaussian) current profiles can also partially flatten the wakefield, but will result in tails in the accelerated energy spectrum.

In this Letter, we experimentally demonstrate optimal beam loading of a nonlinear plasma wakefield, resulting in simultaneous preservation of per-mille energy spreads, high energy-transfer efficiency, and full charge coupling. The optimized acceleration regime was reached by employing quasitrapezoidal trailing bunches and performing a large multidimensional scan of beam and plasma parameters. Direct measurement of field flattening within the trailing bunch was performed with a novel high-resolution wakefieldsampling technique [22]. While the energy gain was modest, preservation of the small initial energy spread implies that the wakefield was flattened at the few-percent level.

The experiment was performed at the FLASHForward plasma-accelerator facility at DESY [23]. Electron bunches were provided by the FLASH linac [24]; generated with a photoelectron gun and accelerated to $1 \mathrm{GeV}$ using superconducting radio-frequency (rf) cavities. The bunches were compressed by two magnetic chicanes and linearized in longitudinal phase space by a third-harmonic cavity. Three energy collimators in a dispersive section were used for 

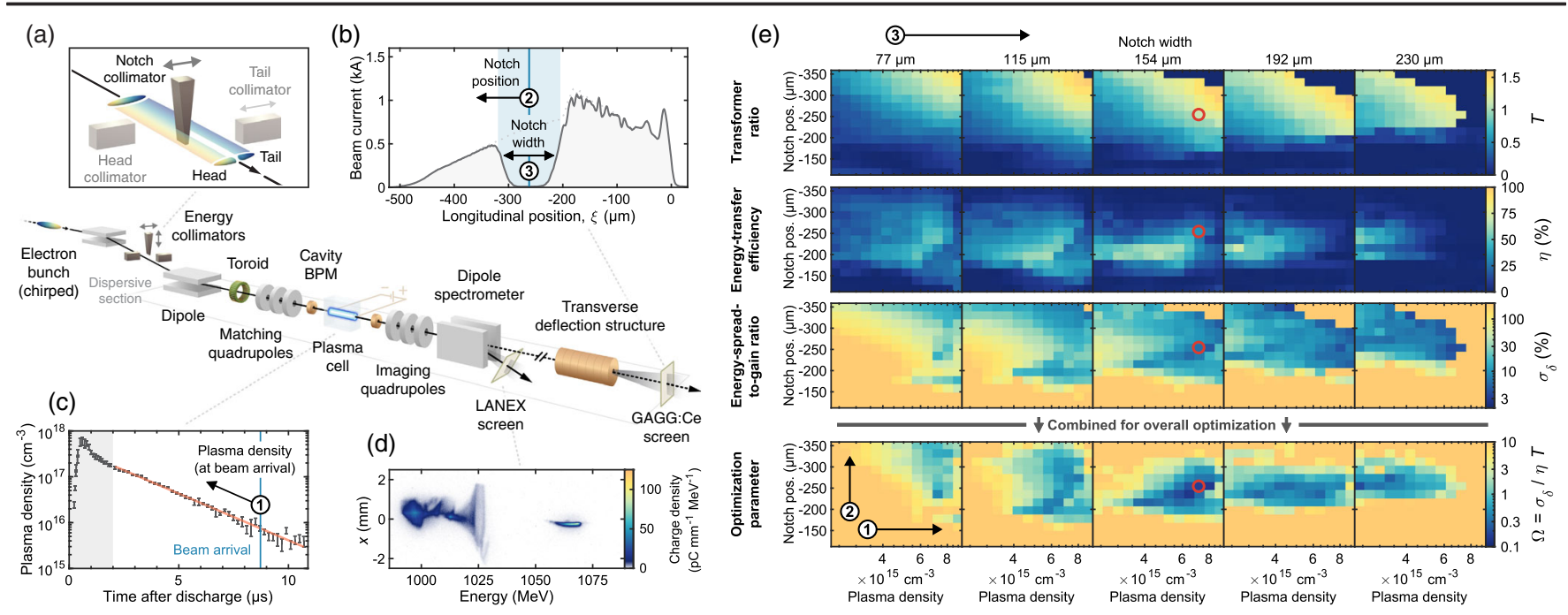

FIG. 1. (a) A notch collimator with an adjustable width and position, located in a dispersive section, was used to create two bunches from a chirped electron bunch. (b) The resulting current profiles were measured with a downstream TDS. (c) A discharge capillary was used to form a plasma channel. The plasma density was measured to decay exponentially (orange trendline) after the initial dischargethe density was varied by adjusting the beam arrival time. Measurements shortly after the discharge (shaded area) may be inaccurate due to temperature effects [28]. (d) Energy spectra were measured with a dipole spectrometer and a set of quadrupoles for point-to-point imaging. (e) 3D parameter scan of plasma density (1) versus notch position (2) as a function of notch width (3). Each row of plots shows, from the top, measurements at each step of the transformer ratio $(T)$, energy-transfer efficiency $(\eta)$ and energy-spread-to-gain ratio $\left(\sigma_{\delta}\right)$, which were combined into an overall optimization parameter $\Omega$ [Eq. (2)]. A full characterization was performed at the optimal operating point (red circle).

detailed shaping of the current profile [25]: low- and high-energy collimators for removing the bunch head and tail, respectively, and a wedge-shaped notch collimator for creating a double-bunch profile with an adjustable separation [Figs. 1(a) and 1(b)]. The bunch charge was measured before and after collimation with toroidal current transformers - the uncollimated charge was $(1018 \pm 1) \mathrm{pC}$. A set of quadrupole magnets was used to tightly focus the electron beam at the entrance of the plasma cell. Two cavitybased beam-position monitors (BPMs) [26], placed directly upstream and downstream of the cell, were used to measure the beam trajectory. The plasma cell consisted of a discharge capillary [27] with a $50 \mathrm{~mm}$-long, $1.5 \mathrm{~mm}$-diameter channel milled from two blocks of sapphire, filled with argon through two gas inlets (placed $2.5 \mathrm{~mm}$ from the ends) at a backing pressure of 20 mbar, and discharged with short (400 ns), high-voltage $(25 \mathrm{kV})$, high-current $(500 \mathrm{~A})$ pulses. The evolution of the plasma density at the cell center [Fig. 1(c)] was measured in an identical setup where the argon was doped with $3 \%$ hydrogen to observe spectral-line broadening of the $\mathrm{H}$-alpha line $[28,29]$. The electron bunches were diagnosed downstream of the plasma cell with a dipole spectrometer, using five quadrupoles for point-to-point imaging of the beam from the plasma exit to a LANEX screen [Fig. 1(d)]. Further downstream, an $\mathrm{X}$-band rf transverse deflection structure (TDS) $[30,31]$ was used to streak the bunch onto a cerium-doped gadolinium aluminium gallium garnet (GAGG:Ce) screen for measurements of the current profile - the full length of the bunch was approximately $500 \mu \mathrm{m}$ with a peak current of $1 \mathrm{kA}$. The
TDS was only operated with non-plasma-interacted bunches and relaxed beam focusing due to the complexity of transporting high-divergence bunches the full distance $(33 \mathrm{~m})$ from the plasma to the TDS measurement screen.

High-quality plasma acceleration requires precise control of the transverse phase space of the incoming beam. The matching quadrupoles were set to focus the beam to a waist close to the plasma entrance with a beta function [32] of approximately $10 \mathrm{~mm}$ in both planes. The waist location and beta function were then measured and fine-tuned with $\mathrm{mm}$ precision using a novel jitter-based measurement technique [33]. An object-plane scan was performed with the imaging quadrupoles, verifying the location of the waist and measuring the horizontal divergence to be $(0.23 \pm$ 0.03 ) $\mathrm{mrad}$ in the tail and up to $1 \mathrm{mrad}$ in the head (higher due to coherent-synchrotron-radiation effects [34]). These measurements imply minimum beam sizes of $2-10 \mu \mathrm{m}$ and normalized slice emittances of 1-20 mm mrad (tail to head, respectively). The vertical divergence could not be measured, but is expected to be similar to the horizontal divergence of the tail. In order to inject charge in the very back of the plasma cavity, the bunch was straightened by adjusting quadrupoles and sextupoles in the dispersive section to cancel beam tilts and curvatures, respectively [35]. A plasma density of approximately $6 \times 10^{15} \mathrm{~cm}^{-3}$ was found to best match the plasma-cavity length to the full (uncollimated) bunch length.

A multidimensional scan of beam and plasma parameters was performed to locate the optimal-beam-loading 
operating point. Three important parameters were identified: (1) the plasma density, adjusted by changing the beam arrival time after the discharge; (2) the longitudinal position of the current-profile notch, adjusted by transverse movements of the wedge-shaped notch collimator; and (3) the width of the notch, adjusted by vertical movements of the notch collimator. While the current profile prior to collimation remained constant, the plasma density was used to change the normalized bunch length (relative to the plasmacavity length), and the two notch parameters were used to change the separation distance and charge ratio between the two bunches. Each parameter was scanned across the full range of values where acceleration could be observed, with a total of $5 \times 13 \times 13$ steps averaged over 15 shots per step; 12675 shots in total.

At each step, three wakefield properties were calculated from the resulting spectra to evaluate the shape of the longitudinally averaged wakefield: the transformer ratio, the energy-transfer efficiency, and the energy-spread-togain ratio. The longitudinally averaged transformer ratio $T$ is calculated as the mean energy gain of the trailing bunch normalized by the maximum energy loss within the driver [36]; the longitudinally averaged energy-transfer efficiency is calculated as

$$
\eta=-\frac{\Delta\langle E\rangle_{\mathrm{acc}} Q_{\mathrm{acc}}}{\Delta\langle E\rangle_{\mathrm{dec}} Q_{\mathrm{dec}}}
$$

where $\Delta\langle E\rangle$ denotes the mean energy change of each bunch, $Q_{\text {acc }}$ is the final accelerated charge, and $Q_{\text {dec }}$ is the average of the initial and final decelerated charge - the best estimate of the wake-driving charge in case of charge loss from the driver. Finally, the energy-spread-to-gain ratio, $\sigma_{\delta}$, is calculated as the full width at half maximum (FWHM) of the accelerated spectrum normalized by the mean energy gain. All three properties $\left(T, \eta\right.$, and $\left.\sigma_{\delta}\right)$ are dimensionless and instantaneous representations of the wakefield, and therefore allow the quality of the beam-loading process to be evaluated independently of acceleration length and gradient.

Figure 1(e) shows the measurement of the three wakefield properties. This complex parameter space has multiple optima based on the desired objective: the highest transformer ratio was measured to be $(1.61 \pm 0.01)$, the highest efficiency was $(71 \pm 4) \%$ (subject to systematic errors discussed below), and the lowest energy-spread-to-gain ratio was $(3.1 \pm 0.2) \%$ FWHM, where the quoted uncertainty represents the root-mean-square (rms) variation at the optimum step. However, a useful operating point requires all properties to be simultaneously optimized. It is therefore helpful to define a new wakefield optimization parameter,

$$
\Omega=\frac{\sigma_{\delta}}{\eta T},
$$

as an overall figure of merit. Minimizing this quantity simultaneously minimizes the energy-spread-to-gain ratio $\sigma_{\delta}$, while maximizing the energy-transfer efficiency $\eta$ and the transformer ratio $T$. Measurements of this optimization parameter show a distinct minimum in the parameter space. A careful characterization was performed at this optimal operating point [red circle in Fig. 1(e)], where the value of $\Omega$ was measured to be $(0.077 \pm 0.012)$-between 1 and 2 orders of magnitude lower than in previous experiments $[17,18]$.

Figure 2(a) shows spectrometer images and spectra for a single shot at the optimal operating point: a $(490 \pm 10) \mathrm{pC}$ driver accelerates a $100 \mathrm{pC}$ trailing bunch while preserving (and slightly dechirping [37-39]) the $0.16 \%$ FWHM initial energy spread. A small negative skewness (i.e., a low-energy tail) is introduced in the accelerated spectrum, caused by imperfections in the trailing-bunch current profile compared to the ideal shape described by Tzoufras et al. [19]. To ensure good energy resolution, the spectrometer was configured to form a point-to-point image for the mean energy of the trailing bunch in spectrum measurements both with and without plasma interaction.
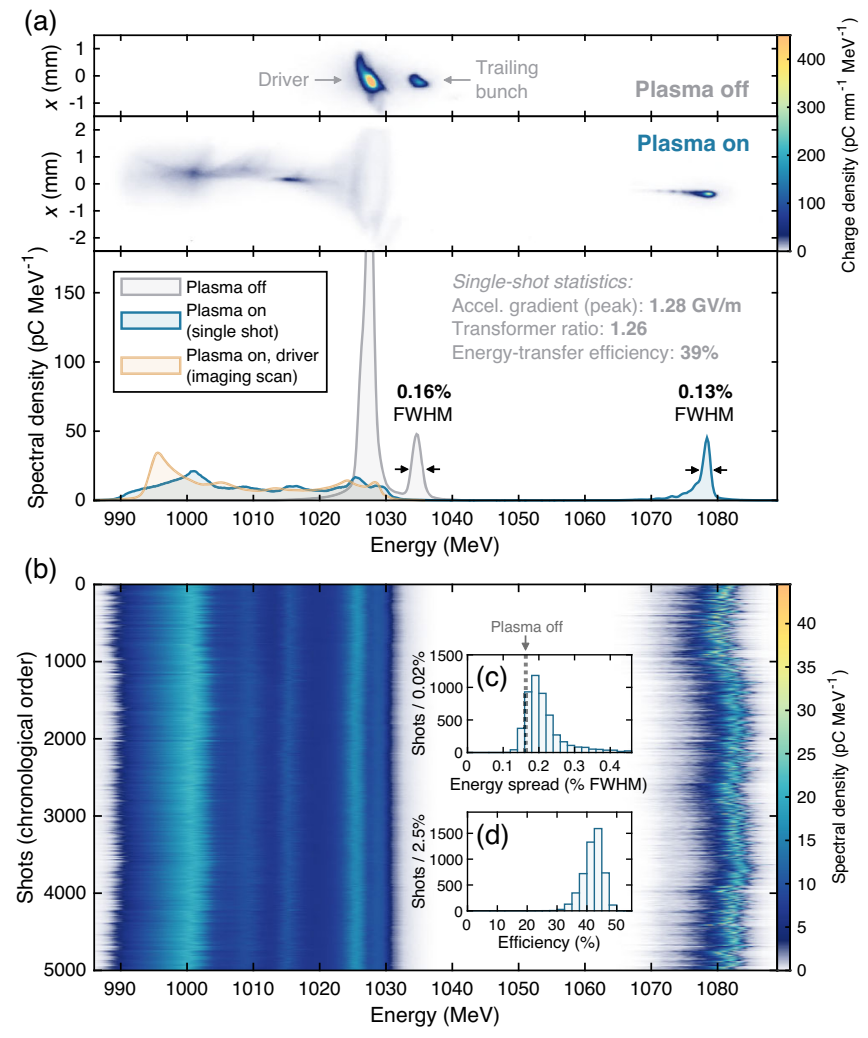

FIG. 2. (a) Spectrometer images at the optimal operating point [red circle in Fig. 1(e)], as well as the corresponding energy spectra, for shots with and without plasma interaction. The initial energy spread of the trailing bunch is preserved. (b) High stability is observed across 5000 consecutive shots-the energy gain is stable to within $3 \%$ rms. (c) In $6.4 \%$ of these shots, the energy spread is lower than or equal to the initial energy spread (dotted line). (d) Simultaneously, high energy-transfer efficiency is observed, distributed between $30 \%$ and $50 \%$. 
A 5000-shot high-statistics dataset [Fig. 2(b)] shows that the energy gain was $(45.4 \pm 1.4) \mathrm{MeV}$ - stable to $3 \% \mathrm{rms}$. The energy spread was fully preserved in $6.4 \%$ of these shots [Fig. 2(c)], while the rest had a median energy spread of $0.2 \%$ FWHM (a relative increase of 28\%), indicating that the optimal operating point is highly sensitive to even lowlevel jitters in beam or plasma parameters.

The energy-transfer efficiency at the optimal operating point was measured to be $(42 \pm 4) \%$ [Fig. 2(d)]. This measurement depends on the accuracy of the charge distribution across the full energy spectrum, which cannot be measured everywhere simultaneously with good resolution-the spectrum is distorted away from the imaging energy due to nonzero divergence and angular misalignments. Therefore, an imaging-energy scan was performed to measure the driver spectrum in $0.5 \%$ energy steps-the resulting spliced spectrum is shown in Fig. 2(a). This leads to an energy-efficiency correction of -3.6 percentage points (already accounted for in the efficiency quoted above) compared to single-shot spectra where only the accelerated bunch is imaged. This systematic effect is expected to be similar for the efficiency measurements in Fig. 1(e). Furthermore, only $94 \%$ of the initial driver charge is measured in the spectrometer. Given that the energy loss of the missing driver charge is unknown, this introduces a systematic uncertainty on the efficiency of \pm 1.3 percentage points.

Energy-spread preservation is only strictly meaningful if no charge is lost. Figure 3 shows a tail-collimator scan of the trailing bunch at the optimal operating point, demonstrating that full charge coupling could be achieved with appropriate collimation of the bunch tail. When the collimator is fully extracted, only $(98 \pm 9)$ pC of the initial $(176 \pm 3) \mathrm{pC}$ of charge is accelerated. As the tail of the trailing bunch gets progressively removed, the accelerated charge remains approximately constant until the incoming charge equals the accelerated charge - this is caused by strong defocusing of the trailing bunch as the plasmasheath electrons return to the axis. Beyond this point, both the incoming and accelerated charge decrease identically, showing an approximately $100 \%$ charge-coupling efficiency. Note that the charge jitter decreases significantly (from $10 \%$ to $4 \% \mathrm{rms}$ ) when transitioning into the full charge-coupling regime, indicating that if the tail of the trailing bunch reaches the back of the plasma cavity, its coupling is highly sensitive to variations of the cavity length (determined by the plasma density and driver parameters).

Direct measurement of the wakefield flattening was performed using a newly developed wakefield-sampling technique (see Ref. [22] for a complete description). The measurement consisted of a tail-collimation scan of the incoming driver and trailing bunch from tail to head, observing the energy spectrum of each slice as they are removed from the overall spectrum. This scan was

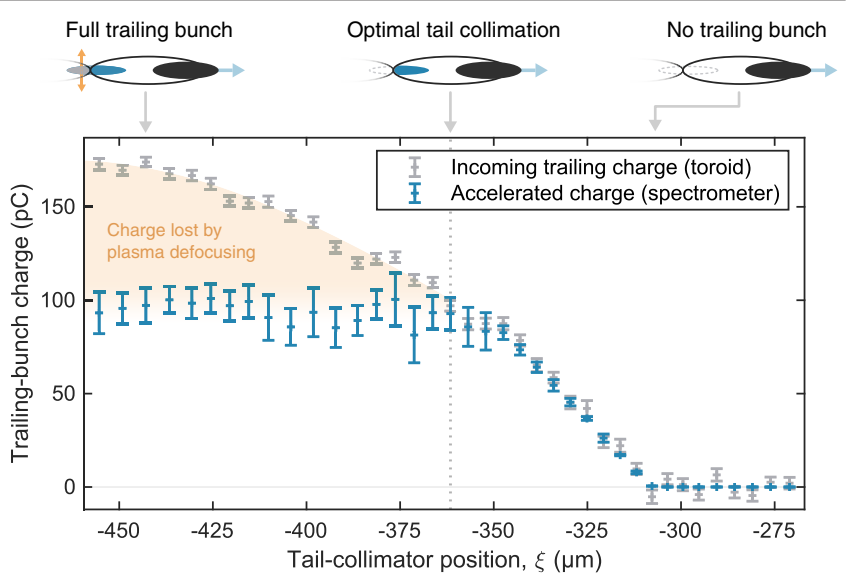

FIG. 3. Comparison of incoming trailing-bunch charge (gray points) and accelerated charge (blue points) in a tail-collimator scan. Charge is lost until position $\xi \lesssim-360 \mu \mathrm{m}$, after which full charge coupling is observed. This transition is caused by the defocusing field of plasma electrons crossing the axis: beam particles behind the axis crossing are lost, whereas particles ahead remain focused. The incoming trailing charge is calculated by subtracting the mean driver charge from the total charge, as measured by a toroid, where the error bars represent the standard error of the mean. The accelerated charge is measured on the spectrometer, where the error bars represent the standard deviation. Data in Fig. 2 were taken at the optimal tail-collimator position (dotted line).

performed both with and without plasma interaction in order to calculate the energy change of each slice, and repeated at the TDS to measure the longitudinal position within the current profile of each collimator position (without plasma interaction to allow the beam to be transported). Figure 4(a) shows the measured wakefield for the optimal operating point, demonstrating that it has been locally flattened by the trailing bunch at $-1.29 \mathrm{GV} / \mathrm{m}$, with a variation of $2.8 \% \mathrm{rms}$ across the $60 \mu \mathrm{m}$ where the signal-to-noise ratio was sufficiently high. For comparison, a measurement was also performed for the full bunch (i.e., no notch collimator) at a slightly lower density. Calculating the longitudinally averaged wakefield from the overall energy gain requires knowledge of the plasma length. Since the longitudinal plasma density profile is not known in detail, an effective length of $34.2 \mathrm{~mm}$ is assumed, slightly shorter than the distance between the two gas inlets $(39 \mathrm{~mm})$.

Beam loading can be demonstrated indirectly by comparison of the measured wakefield with an unloaded wakefield from a particle-in-cell (PIC) simulation. For this, accurate modeling of the plasma acceleration process is required. Using longitudinal-phase-space measurements from the TDS and transverse-phase-space measurements from the spectrometer and the BPMs [33], a detailed reconstruction of the $6 \mathrm{D}$ beam phase space was possible. The vertical slice emittance was the only parameter that could not be measured and was assumed to be similar to the 


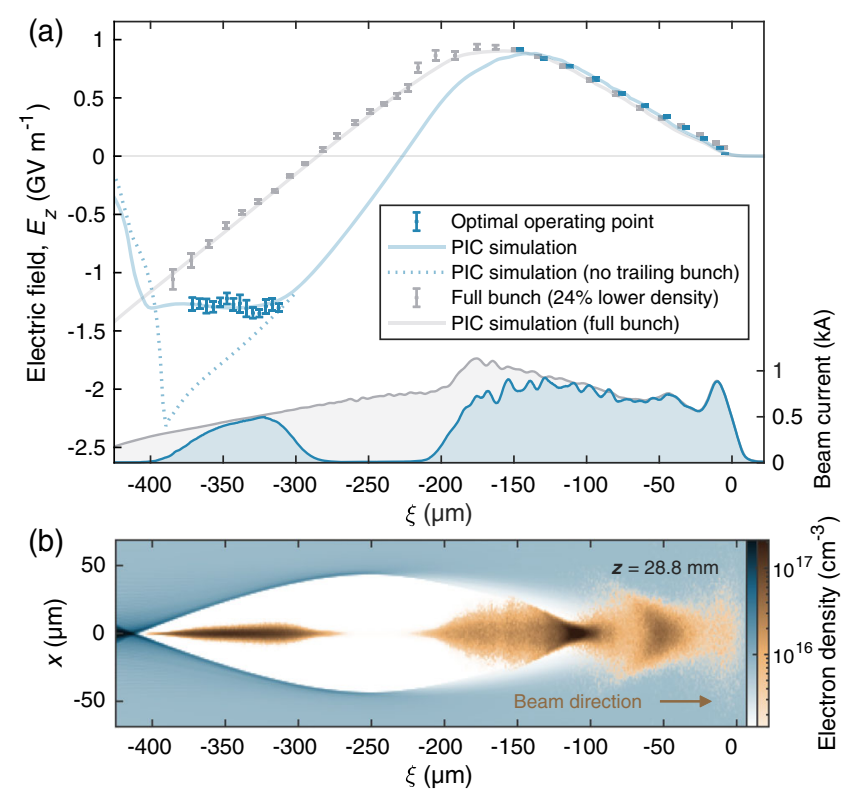

FIG. 4. (a) Longitudinally averaged wakefield measured using a tail-collimator scan, both for optimal beam loading (blue points) and for a full bunch (gray points) - in excellent agreement with PIC simulations (blue and gray solid lines). A simulation with no trailing bunch (blue dotted line) indicates that the wakefield was flattened by beam loading. The beam currents of the collimated and the full bunch (blue and gray areas) were measured with a TDS. (b) Snapshot showing cross sections of beam (orange) and plasma electron density (blue) for a simulation of the optimal operating point, on a logarithmic color scale. The simulated flattop plasma density is $7.2 \times 10^{15} \mathrm{~cm}^{-3}$.

horizontal slice emittance of the tail. A flattop plasma density $4 \%$ lower than the measured central density [Fig. 1(c)] was found to best match the wakefield measurement, suggesting that any density ramps present had an effect small enough to justify a flat-top model. Simulations were performed with the 3D quasistatic code HiPACE [40] in a grid of $512 \times 512 \times 512$ cells, 4 plasma particles per cell in the wake region, a spatial resolution of $1.18 \mu \mathrm{m}$ in all dimensions, $4.2 \times 10^{6}$ beam particles, and a time step of $5 \omega_{p}^{-1}$, where $\omega_{p}$ is the angular plasma frequency. The simulated longitudinally averaged wakefield is consistent with the sampling measurement for both the optimal operating point and the full bunch. Repeating the optimaloperating-point simulation without a trailing bunch clearly shows that the unloaded wakefield would not have been flat, and that strong beam loading was needed to flatten the field-consistent with the high energy-transfer efficiency observed in the measurement. Simulations indicate that while the initial energy spread would also be preserved for trailing bunches with a similar Gaussian current profile, the accelerated spectrum would have longer tails compared to the quasitrapezoidal bunches used in the experiment. The spread in wakefield amplitude across the trailing bunch (weighted by charge) was reduced by approximately $40 \%$ (from $9.3 \%$ to $5.8 \%$ rms) as a result of this current-profile shaping.

In conclusion, we have experimentally demonstrated optimal beam loading in a plasma-wakefield accelerator. Optimization of the combined wakefield parameter $\Omega$ [Eq. (2)] resulted in simultaneous preservation of per-mille energy spreads, $(42 \pm 4) \%$ energy-transfer efficiency and full charge coupling for $100 \mathrm{pC}$ bunches accelerated with high stability $(3 \% \mathrm{rms})$ at a gradient of $1.3 \mathrm{GV} / \mathrm{m}$-all in excellent agreement with simulations. This represents a major step towards precise and application-relevant plasma-wakefield accelerators. Reaching per-mille-level control of the wakefield will enable energy-spread preservation also for larger energy gains, which, combined with emittance preservation, can open the door to a new generation of free-electron lasers and particle colliders.

The authors would like to thank M. Dinter, S. Karstensen, S. Kottler, K. Ludwig, F. Marutzky, A. Rahali, V. Rybnikov, A. Schleiermacher, the FLASH management, and the DESY FH and M divisions for their scientific, engineering and technical support. This work was supported by Helmholtz ARD and the Helmholtz IuVF ZT-0009 programme, as well as the Maxwell computational resources at DESY.

*carl.a.lindstroem@desy.de

'Present address: LOA, ENSTA ParisTech, CNRS, Ecole Polytechnique, Université Paris-Saclay, 91762 Palaiseau, France.

[1] T. Tajima and J. M. Dawson, Laser Electron Accelerator, Phys. Rev. Lett. 43, 267 (1979).

[2] P. Chen, J. M. Dawson, R. W. Huff, and T. Katsouleas, Acceleration of Electrons by the Interaction of a Bunched Electron Beam with a Plasma, Phys. Rev. Lett. 54, 693 (1985).

[3] R. D. Ruth, A. W. Chao, P. L. Morton, and P. B. Wilson, A plasma wake field accelerator, Part. Accel. 17, 171 (1985).

[4] M. J. Hogan, C. D. Barnes, C. E. Clayton, F. J. Decker, S. Deng, P. Emma et al., Multi-GeV Energy Gain in a PlasmaWakefield Accelerator, Phys. Rev. Lett. 95, 054802 (2005).

[5] I. Blumenfeld, C. E. Clayton, F.-J. Decker, M. J. Hogan, C. Huang, R. Ischebeck et al., Energy doubling of $42 \mathrm{GeV}$ electrons in a metre-scale plasma wakefield accelerator, Nature (London) 445, 741 (2007).

[6] S. Corde, E. Adli, J. Allen, W. An, C. I. Clarke, B. Clausse et al., High-field plasma acceleration in a high-ionizationpotential gas, Nat. Commun. 7, 11898 (2016).

[7] C. Joshi and T. Katsouleas, Plasma accelerators at the energy frontier and on tabletops, Phys. Today 56, 6, 47 (2003).

[8] E. Adli, J.-P. Delahaye, S. J. Gessner, M. J. Hogan, T. O. Raubenheimer, W. An, C. Joshi, and W. B. Mori, A beam driven plasma-wakefield linear collider: From Higgs factory to multi-TeV, SLAC Report No. SLAC-PUB-15426, 2013. 
[9] M. E. Couprie, A. Loulergue, M. Labat, R. Lehe, and V. Malka, Towards a free electron laser based on laser plasma accelerators, J. Phys. B 47, 234001 (2014).

[10] A. R. Maier, M. Kirchen, and F. Grüner, Brilliant light sources driven by laser-plasma accelerators, Synchrotron Light Sources and Free-Electron Lasers: Accelerator Physics, Instrumentation and Science Applications (Springer, Cham, 2020), p. 245.

[11] J. M. J. Madey, Stimulated emission of bremsstrahlung in a periodic magnetic field, J. Appl. Phys. 42, 1906 (1971).

[12] The International Linear Collider Technical Design Report, edited by T. Behnke et al., Volume 1: Executive Summary (2013).

[13] S. van der Meer, Improving the power efficiency of the plasma wakefield accelerator, CLIC Note No. 3, 1985.

[14] T. Katsouleas, S. Wilks, P. Chen, J. M. Dawson, and J. J. Su, Beam loading in plasma accelerators, Part. Accel. 22, 81 (1987).

[15] P. Chen, J. J. Su, J. M. Dawson, K. L. F. Bane, and P. B. Wilson, Energy Transfer in the Plasma Wake-Field Accelerator, Phys. Rev. Lett. 56, 1252 (1986).

[16] K. V. Lotov, Efficient operating mode of the plasma wakefield accelerator, Phys. Plasmas 12, 053105 (2005).

[17] M. Litos, E. Adli, W. An, C. I. Clarke, C. E. Clayton, S. Corde et al., High-efficiency acceleration of an electron beam in a plasma wakefield accelerator, Nature (London) 515, 92 (2014).

[18] M. Litos, E. Adli, J. M. Allen, W. An, C. I. Clarke, S. Corde et al., $9 \mathrm{GeV}$ energy gain in a beam-driven plasma wakefield accelerator, Plasma Phys. Controlled Fusion 58, 034017 (2016).

[19] M. Tzoufras, W. Lu, F. S. Tsung, C. Huang, W. B. Mori, T. Katsouleas, J. Vieira, R. A. Fonseca, and L. O. Silva, Beam Loading in the Nonlinear Regime of Plasma-Based Acceleration, Phys. Rev. Lett. 101, 145002 (2008).

[20] J. B. Rosenzweig, Nonlinear Plasma Dynamics in the Plasma Wake-Field Accelerator, Phys. Rev. Lett. 58, 555 (1987).

[21] W. Lu, C. Huang, M. Zhou, W. B. Mori, and T. Katsouleas, Nonlinear Theory for Relativistic Plasma Wakefields in the Blowout Regime, Phys. Rev. Lett. 96, 165002 (2006).

[22] S. Schröder, C. A. Lindstrøm, G. Boyle, R. D’Arcy, S. Diedrichs, M. J. Garland et al., High-resolution sampling of beam-driven plasma wakefields, Nat. Commun. 11, 5984 (2020).

[23] R. D’Arcy, A. Aschikhin, S. Bohlen, G. Boyle, T. Brümmer, J. Chappell et al., FLASHForward: Plasma wakefield accelerator science for high-average-power applications, Phil. Trans. R. Soc. A 377, 20180392 (2019).

[24] S. Schreiber and B. Faatz, The free-electron laser FLASH, High Power Laser Sci. Eng. 3, e20 (2015).

[25] S. Schröder, K. Ludwig, A. Aschikhin, R. D’Arcy, M. Dinter, P. Gonzalez et al., Tunable and precise two-bunch generation at FLASHForward, J. Phys. Conf. Ser. 1596, 012002 (2020).

[26] D. Lipka, Cavity BPM designs, related electronics and measured performances, Proceedings of DIPAC'09, Basel, Switzerland, 2009 (CERN, Geneva, 2009), p. 280.
[27] D. J. Spence and S. M. Hooker, Investigation of a hydrogen plasma waveguide, Phys. Rev. E 63, 015401(R) (2000).

[28] M. A. Gigosos, M. A. González, and V. Cardeñoso, Computer simulated Balmer-alpha, -beta and -gamma Stark line profiles for non-equilibrium plasmas diagnostics, Spectrochim. Acta B Atom. Spectros. 58, 1489 (2003).

[29] J. M. Garland, G. Tauscher, S. Bohlen, G. J. Boyle, R. D’Arcy, L. Goldberg, K. Põder, L. Schaper, B. Schmidt, and J. Osterhoff, Evolution of longitudinal plasma-density profiles in discharge capillaries for plasma wakefield accelerators, arXiv:2010.02567.

[30] A. Grudiev, Design of compact high power RF components at X-band, CLIC Note No. 1067, 2016.

[31] R. D’Arcy, A. Aschikhin, P. Gonzalez Caminal, J. Osterhoff, and V. Libov, Longitudinal phase space reconstruction at FLASHForward using a novel X-band transverse deflection cavity (XTDC), Proceedings of IPAC'18, Vancouver, Canada (JACoW, Geneva, 2018), p. 1567.

[32] E. D. Courant and H. S. Snyder, Theory of the alternatinggradient synchrotron, Ann. Phys. (N.Y.) 3, 1 (1958).

[33] C. A. Lindstrøm, R. D’Arcy, M. J. Garland, P. Gonzalez, B. Schmidt, S. Schröder, S. Wesch, and J. Osterhoff, Matching small $\beta$ functions using centroid jitter and two beam position monitors, Phys. Rev. Accel. Beams 23, 052802 (2020).

[34] E. L. Saldin, E. A. Schneidmiller, and M. V. Yurkov, On the coherent radiation of an electron bunch moving in an arc of a circle, Nucl. Instrum. Methods Phys. Res., Sect. A 398, 373 (1997).

[35] M. W. Guetg, B. Beutner, E. Prat, and S. Reiche, Optimization of free electron laser performance by dispersionbased beam-tilt correction, Phys. Rev. Accel. Beams 18, 030701 (2015).

[36] G. Loisch, G. Asova, P. Boonpornprasert, R. Brinkmann, Y. Chen, J. Engel et al., Observation of High Transformer Ratio Plasma Wakefield Acceleration, Phys. Rev. Lett. 121, 064801 (2018).

[37] R. D’Arcy, S. Wesch, A. Aschikhin, S. Bohlen, C. Behrens, M.J. Garland et al., Tunable Plasma-Based Energy Dechirper, Phys. Rev. Lett. 122, 034801 (2019).

[38] V. Shpakov, M. P. Anania, M. Bellaveglia, A. Biagioni, F. Bisesto, F. Cardelli et al., Longitudinal Phase-Space Manipulation with Beam-Driven Plasma Wakefields, Phys. Rev. Lett. 122, 114801 (2019).

[39] Y. P. Wu, J. F. Hua, Z. Zhou, J. Zhang, S. Liu, B. Peng et al., Phase Space Dynamics of a Plasma Wakefield Dechirper for Energy Spread Reduction, Phys. Rev. Lett. 122, 204804 (2019).

[40] T. Mehrling, C. Benedetti, C. B. Schroeder, and J. Osterhoff, HiPACE: a quasi-static particle-in-cell code, Plasma Phys. Controlled Fusion 56, 084012 (2014).

Correction: The byline footnotes for the first and ninth author were improperly set with the wrong footnote indicators and positioned out of order during the production process and have been set right. The author names in the citation of other work in the abstract given in the HTML version dropped out during the production process and has been fixed. The PDF version was set properly. 